\title{
ESTILOS DE APRENDIZAGEM. UM MODELO DE ESCALA DE OBSERVAÇÃO DOCENTE PARA O ESTILO DE APRENDIZAGEM - REApt -
}

\author{
ESTILOS DE APRENDIZAJE. UN MODELO DE ESCALA DE OBSERVACIÓN \\ DOCENTE PARA EL ESTILO DE APRENDIZAJE -REApt-
}

\author{
LEARNING STYLES. A MODEL OF TEACHER OBSERVATION SCALE FOR \\ LEARNING STYLE
}

\author{
Eladio SEBASTIAN-HEREDERO ${ }^{1}$
}

RESUMO: O trabalho apresenta o resultado de uma pesquisa sobre os estilos de aprendizagem e a busca de um instrumento para seu conhecimento por professores do ensino básico. Os estilos de aprendizagem têm sido muito estudados nos últimos cinquenta anos, tornando-se objeto de múltiplas investigações e atualizações com base nas correntes psicológicas (HERRMANN, 1982) e pedagógicas (GARDNER, 1995). Da mesma forma, existem vários instrumentos (LSI; HBDI; LSI++; CHAEA) que medem os estilos de aprendizagem obedecendo a esses mesmos princípios. Como fruto deste estudo nasce o REA, que aqui apresentamos com sua fundamentação teórica (KOHLBER, 1969; BANDLER e GRINDER, 1979; KOLB, 1984; DUNN, DUNN e PRICE, 1985; FELDER e SILVERMAN, 1988), adaptado a contextos escolares ordinários, que pode ser completado por professores como instrumento de reflexão sobre seus educandos e, ao mesmo tempo, como ilustrador de novas formas para entender como funciona a forma de aprender dos alunos e, assim, intervir nas aulas.

PALAVRAS-CHAVE: Estilos de aprendizagem. Atenção à diversidade. Inclusão escolar.

RESUMEN: Presentamos el resultado de una investigación sobre los estilos de aprendizaje y búsqueda de un instrumento para detección por profesores de enseñanza básica. Los estilos de aprendizaje han sido estudiados con gran profundidad en los últimos cincuenta años en base a corrientes psicológicas (HERRMANN, 1982), pedagógicas (GARDNER, 1995) y teorías de aprendizaje. Así mismo existen varios instrumentos (LSI; HBDI; LSI++; CHAEA) que miden los estilos y que obedecen a estos mismos referentes. Con este estudio creamos el REA con una fundamentación teórica integradora de diferentes teorías (KOHLBER, 1969; BANDLER y GRINDER, 1979; KOLB, 1984; DUNN, DUNN y PRICE, 1985; FELDER y SILVERMAN, 1988), adaptada a contextos escolares ordinarios, que puede ser completada por profesores como instrumento de reflexión sobre sus educandos y, al mismo tiempo, como ilustradora de nuevas formas de entender cómo funciona el aprender de los alumnos y así intervenir en las aulas.

${ }^{1}$ Universidad de Alcalá (UAH), Madrid - Espanha. Profesor Doctor de la Universidad de Alcalá (España) y Profesor colaborador de la UNESP (Brasil). Profesor investigador de la UNIARA (Brasil). Profesor de la Consejería de Educación de Castilla La Mancha en Guadalajara (España). ORCID: https://orcid.org/0000-00030293-4395. E-mail: eladio.sebastian@gmail.com 
PALABRAS CLAVE: Estilos de aprendizaje. Atención a la diversidad. Inclusión escolar.

ABSTRACT: This work is the result of a research on learning styles and search for an instrument for detection by teachers of basic education. Learning styles have been studied with great depth in the last fifty years based on psychological currents (HERRMANN, 1982) or pedagogical (GARDNER, 1995) and learning theories. There are also several instruments (LSI, HBDI, LSI ++, CHAEA) that measure the styles and that obey these same referents. With this study we created the -REA- with a theoretical foundation integrating different theories (KOHLBER, 1969, BANDLER and GRINDER, 1979, KOLB, 1984, DUNN, DUNN and PRICE, 1985, FELDER and SILVERMAN, 1988), adapted to ordinary school contexts, that can be completed by teachers as an instrument of reflection on their students and, at the same time, as an illustrator of new ways of understanding how students learn and intervention in classrooms.

KEYWORDS: Learning styles. Attention to diversity. School inclusion

\section{Introdução}

Os seres humanos, por natureza, são capazes de aprender em qualquer situação e isso depende de vários fatores. Começaremos por reconhecer que cada pessoa é única e tem inteligências diferentes. Gardner (1995) advertiu que o Coeficiente Intelectual (CI) não é a única forma de inteligência que existe, e identificou e descreveu até oito tipos de inteligências distintas. Segundo esta concepção da mente humana, existe vários tipos de capacidades mentais e, portanto, diferentes formas de aprender utilizando as competências de cada pessoa. Isso levou à conclusão de que se há vários tipos de inteligências, existem também vários tipos ou estilos de aprendizagem em função de como se desenvolvem as mesmas em cada pessoa, o que torna imprescindível reconhecer quando falamos de diversidade de alunos e de escola inclusiva.

$\underline{\text { Kolb }}$ (1984) chegou à conclusão que a aprendizagem se desenvolvia a partir de três fatores causais: fatores genéticos, ou seja, por herança; fatores derivados das experiências da vida, portanto, modificáveis em função do que fazemos e praticamos, incluídas as aprendizagens escolares e os fatores derivados das experiências do entorno.

Também existem explicações científicas ligadas com o cérebro e como este utiliza as diversas formas de operar, de pensar, de criar ou de aprender, sendo este o modelo dos Quadrantes Cerebrais de Herrmann (1982), segundo o qual cada zona do cérebro utilizada representa uma forma de aprender:

- Zona Cortical Esquerda (Ce): Perfil Perito. Representa uma pessoa lógica- analítica

RIAEE - Revista Ibero-Americana de Estudos em Educação, Araraquara, v. 14, n. 4, p. 2301-2317, out./dez. 2019. E-ISSN: $1982-5587$. DOI: https://doi.org/10.21723/riaee.v14i4.12384 
com uma forma de raciocinar baseado em fatos. Está mais próximo do quantitativo.

- Zona Límbica Esquerda (Le): Perfil Organizador. Pode ser uma pessoa organizada, sequencial e planejada. É muito detalhista.

- Zona Límbica Direita (Ld): Perfil Comunicador. Trata-se de uma pessoa emotiva e com sentimentos com capacidade de estabelecer boas relações interpessoais.

- Zona Cortical Direita (Cd): Perfil Estrategista. É um sujeito holístico, que vê o todo e, portanto, intuitivo e sintetizador. É integrador.

O modelo de Felder e Silverman (1988) é mais prático e classifica os estilos de aprendizagem a partir de cinco dimensões ligadas a atuações do sujeito, as quais estão relacionadas com as respostas que se podem obter nas seguintes perguntas:

- Que tipo de informação recebem preferencialmente os estudantes?

- Através de qual modalidade sensorial é mais efetivamente recebida a informação cognitiva?

- Com que tipo de organização da informação está mais confortável o estudante na hora de trabalhar?

- Como progride o estudante em sua aprendizagem?

- Como o estudante prefere processar a informação?

Existem também os modelos que explicam os estilos de aprendizagem a partir de como os estudantes preferem receber a informação, quer por via visual, auditiva ou manipulativa, inclusive autores que incorporam uma entrada da informação multimodal.

Tudo isso nos leva a concluir que os estilos de aprendizagem estão diretamente relacionados com o tipo de inteligência de cada sujeito e, além disso, de um lado, existem diferentes formas de entender, classificar e determinar os estilos de aprendizagem e, de outro lado, devemos deixar claro que os estilos de aprendizagem são modificáveis e educáveis devido às experiências vividas e ao contexto onde estes se desenvolvem.

Por isso, é necessário que o docente que queira trabalhar na inclusão de todos os seus alunos disponha de estratégias que lhe permitam identificar o estilo de aprendizagem dos mesmos, para poder organizar atividades especificas que permitam favorecer $o$ desenvolvimento das capacidades que cada aluno tem e, dessa forma, melhorar o seu aprendizado. 


\section{Os estilos de aprendizagem}

Keefe (1988) define os estilos de aprendizagem como traços cognitivos, afetivos e fisiológicos relativamente estáveis de como os alunos executam interações e respondem em um contexto de aprendizagem, ou seja, as condições educativas sobre as quais um estudante provavelmente irá aprender mais e melhor.

Insistimos na sua importância desde o ponto de vista pedagógico, pois servem para tratar a organização das sequências didáticas personalizadas em função dos estilos de aprendizagem. Isto é uma forma de resposta de adversidade e uma reflexão para as escolas inclusivas. Existem muitas classificações de estilos de aprendizagem. O seguinte quadro resume algumas das mais importantes.

Quadro 1 - Modelos de Estilos de Aprendizagem

\begin{tabular}{|c|c|c|}
\hline Modelo / Nome & Autor e ano & Tipos de estilo \\
\hline Interação Social na aprendizagem. & Kohlber (1969) & $\begin{array}{l}\text { Competitivo } \\
\text { Evasivo } \\
\text { Colaborativo } \\
\text { Dependente } \\
\text { Independente }\end{array}$ \\
\hline $\begin{array}{l}\text { Modelo da Programação } \\
\text { Neurolinguística- VAK }\end{array}$ & Bandler e Grinder (1979) & $\begin{array}{l}\text { Visual } \\
\text { Auditivo } \\
\text { Cinestésico }\end{array}$ \\
\hline $\begin{array}{l}\text { Modo de processamento da } \\
\text { informação. }\end{array}$ & $\begin{array}{l}\text { Kolb (1984) } \\
\text { Alonso, Gallego e Honey } \\
\text { (1995) }\end{array}$ & $\begin{array}{l}\text { Ativo } \\
\text { Reflexivo } \\
\text { Crítico } \\
\text { Pragmático } \\
\end{array}$ \\
\hline Estímulos de aprendizagem & Dunn, Dunn (1978) & $\begin{array}{l}\text { Ambiente } \\
\text { Emoções } \\
\text { Necessidades sociológicas } \\
\text { Necessidades físicas } \\
\text { Necessidades psicológicas }\end{array}$ \\
\hline Preferências de aprendizagem. & Felder e Silverman (1988) & $\begin{array}{l}\text { Ativo vs Reflexivo } \\
\text { Sensorial vs Intuitivo } \\
\text { Visual vs verbal } \\
\text { Sequencial vs Global } \\
\end{array}$ \\
\hline Tipo de inteligência. & Gardner (1995) & $\begin{array}{l}\text { Lógico-matemático } \\
\text { Linguístico-verbal } \\
\text { Cinestésico } \\
\text { Espacial } \\
\text { Musical } \\
\text { Interpessoal } \\
\text { Intrapessoal } \\
\text { Naturalista }\end{array}$ \\
\hline $\begin{array}{l}\text { Quadrante cerebral que } \\
\text { utiliza na aprendizagem }\end{array}$ & Herrmann (1982) & $\begin{array}{l}\text { Cortical esquerdo } \\
\text { Límbica esquerdo } \\
\text { Cortical direito } \\
\text { Límbica direito }\end{array}$ \\
\hline
\end{tabular}

Fonte: Elaborado pelo autor. 
Explicaremos, a seguir, alguns dos modelos para seu melhor conhecimento e aprofundamento:

\section{a. Classificação: Interação social de Kohlber (1969)}

Segundo este autor, cada pessoa é um universo, e sua forma de se relacionar com os demais também é; isso implica dizer que há diferentes formas de se relacionar com o aprender, tais como: competitivo, evasivo, colaborativo, dependente ou independente. Poderíamos considerar que esta foi a primeira classificação concebida, a saber:

a.1 - Competitivo: Aprendem o material para se sentir melhor que os demais. Competem com seus companheiros e amigos para ver quem obtém a melhor qualificação. Tendem a captar a atenção do professor, necessitam se sentir protagonistas.

a.2 - Evasivo: Ocorre em alunos que não estão interessados em um conteúdo concreto, ou possivelmente em todo o curso. Não costumam participar com o professor, tampouco com seus amigos. Não sentem o que estão aprendendo como algo que devam fazer, não sentem motivação.

a.3 - Colaborativo: Típico de pessoas que querem compartilhar ideias e conhecimentos. Cooperam tanto com os Mestres como com seus próprios companheiros, sentem a aprendizagem como algo interessante e desejam que seus companheiros sintam o mesmo que eles.

a.4 - Dependente: Enxergam os professores e seus companheiros como apoios, necessitam de figuras de autoridade para que lhes digam o que devem fazer. Necessitam que o professor lhes guie constantemente em cada passo que tenham que dar.

a.5 - Independente: São muito autônomos, ainda que sigam confiando em seus professores e companheiros, mesmo assim, seu pensamento é muito mais importante que o dos demais, eles necessitam entender o quê eles querem aprender.

\section{b. Classificação de Kolb (1984)}

É um clássico, uma categorização muito utilizada e faz uma descrição de quatro formas de enfrentar o trabalho por parte dos alunos.

b.1 - Ativos: as habilidades predominantes em pessoas assimiladoras estão relacionadas com a abstração e os estudos teóricos. São práticos e buscam solução aos problemas. Costumam mostrar interesses pela tecnologia. 
b.2 - Reflexivos, Divergentes: querem conhecer e analisar diferentes pontos de vista. Têm uma mente aberta e refletem antes de tomar decisões. Sempre estão dispostos a receber retroalimentação, gostam de escutar. São emocionais e criativos, mostrando interesse pelas artes.

b.3 - Teóricos: preferem ler, estudar, e trabalhar de forma individual, não são especialmente sociáveis. Mostram-se mais interessados nas ideias abstratas do que nas pessoas e nos sentimentos. Não se preocupam com aplicação prática da teoria e necessitam de explicações teóricas claras.

b.4 - Pragmáticos: Guiam-se pela sua intuição, atuando e decidindo sem muita reflexão prévia. São ativos impacientes, frequentemente empregando o enfoque de ensaio-erro. Mostram interesse pelo trabalho em grupo.

Posteriormente, Alonso, Gallego e Honey (1995) fizeram na Espanha uma revisão e trabalharam sobre a base de Kolb (1984), descrevendo estes mesmos quatro estilos: ativo, reflexivo, teórico e prático.

\section{c. Classificação VAK de Bandler e Grinder (1979)}

Segundo a forma de entrada da informação, alguns pesquisadores se interessaram por este fenômeno, antes de Bandler e Grinder (1979); já o descreveram Dunn e Dunn (1978) e, posteriormente, o utilizam também Dunn, Dunn e Price (1985). Todos eles entendem que cada estilo de aprendizagem utiliza uma ou diferentes formas:

c.1 - Visual: são pessoas organizadas e observadoras quando se refere a detalhes. Memorizam coisas mediante a utilização de imagens e podem se concentrar em algo específico, ainda que tenha presença de ruídos. Preferem ler a escutar. Aprendem e lembram observando. Aprendem realizando esquemas, resumos, imagens em geral.

c.2 - Auditivo, é uma pessoa com facilidade para conversação e para aprender línguas.

Tem facilidade para repetir o que escuta e memoriza sequências ou procedimentos. Tem dificuldade em se concentrar se tem ruídos ou sons alheios. Reflete, se expressa e usa a retórica.

c.3 - Cinestésico ou manipulativo: é uma pessoa que se expressa muito corporalmente. É bom em tudo o que tenha atividades ou práticas em geral. Prefere escrever e atuar. Se está estudando ou em um processo de aprendizagem, o faz manipulando, experimentando e fazendo. Precisa de uma abordagem funcional e/ou vivencial.

Existem alguns autores, como Kress e Van Leeuwen (2001), que ao VAK têm acrescentado o multimodal, uma mistura dos anteriores. 


\section{d. Estímulos de aprendizagem de Dunn, Dunn (1978)}

Este modelo é muito completo e foi amplamente utilizado desde 1975. Se fundamenta na crença de que existem cinco grandes estímulos que seriam os que explicariam as diferentes formas de aprender dos alunos.

Quadro 2 - Elementos e estímulos de aprendizagem segundo Dunn e Dunn (1978)

\begin{tabular}{|c|c|c|c|c|c|c|c|}
\hline ESTÍMULOS & \multicolumn{7}{|c|}{ ELEMENTOS } \\
\hline \multirow{3}{*}{$\begin{array}{r}\text { Medio ambiente } \\
\text { Emocional } \\
\text { Sociológico }\end{array}$} & Sonido & & Luz & \multicolumn{2}{|c|}{ Temperatura } & \multicolumn{2}{|c|}{ Diseño } \\
\hline & Motivació & \multicolumn{2}{|r|}{ Persistencia } & \multicolumn{2}{|c|}{ Responsabilidad } & \multicolumn{2}{|c|}{ Estructura } \\
\hline & Uno mismo & Par & Amigos & Equipo & Adu & & Variedad \\
\hline \multirow{2}{*}{$\begin{array}{l}\text { Fisiológico } \\
\text { Psicológico }\end{array}$} & Percepció & & Alimento & \multicolumn{2}{|c|}{ Tiempo } & \multicolumn{2}{|c|}{ Movimiento } \\
\hline & \multicolumn{2}{|c|}{ Global Analitico } & \multicolumn{2}{|c|}{ Hemisferios } & \multicolumn{2}{|c|}{ Impulsivo } & Reflexivo \\
\hline
\end{tabular}

Fonte: Lozano (2000, p. 63)

d.1 - Meio Ambiente: faz referência à influência dos sons, a luz, a temperatura. O desenho e organização da aula na forma de aprender.

d.2 - Emocional: como eles se vêm. Como estão os estímulos de motivação, de ajuda para a persistência no trabalho, a responsabilidade ou a estrutura do centro educativo, neste momento relativo às normas e sua implementação, e sua influência na aprendizagem.

d.3 - Sociológico: estão relacionadas a como o aluno prefere trabalhar, sozinho ou em duplas, em pequenos grupos de amigos, em equipe, sob a supervisão de um adulto ou de diferentes formas.

d.4 - Fisiológico: no aspecto das preferências perceptivas, coincidente com o VAK, mas também se está bem alimentado, se tem tempo suficiente para fazer as tarefas, se é mais ou menos ativo.

d.5 - Psicológico: mais pessoais e que pretendem conhecer como o aluno se organiza para aprender, se é analítico ou global, se é impulsivo ou reflexivo em função e também do hemisfério cerebral que utiliza. Algo próximo às ideias de Hermann (1999).

Esta teoria foi revisada várias vezes, como afirmam Dunn, Dunn y Price (1985). 


\section{e. O modelo de Felder e Silverman (1988)}

Trabalha sobre três aspectos diferentes: o que é significativo para o estudante que aprende, os estilos de aprendizagem preferidos e as estratégias utilizadas. Desta maneira eles definem quatro tipos de estilos em forma de pares para facilitar o seu reconhecimento:

e.1 - Ativo ou Reflexivo, ou seja, a forma de enfrentar a tarefa;

e.2 - Sensorial ou Intuitivo, ou seja, formas de como o aluno aprende;

e.3 - Visual ou verbal, como forma de entrada da informação;

e. 4 - Sequencial ou global, sobre a forma em que integra os conhecimentos.

\section{f. Gardner (1995) e as inteligências múltiplas:}

Ainda que não se trate em si mesmo de estilos de aprendizagem, podemos correlacionar diretamente o tipo de inteligência dos alunos com determinadas preferências em que e como aprender. Assim, os oito tipos de inteligência que descreve nos servem para entender as habilidades que os aprendizes têm.

f.1 - Lógico-matemático: a habilidade para pensar de forma racional e lógica;

f.2 - Linguístico-verbal: a habilidade para utilizar a linguagem de forma criativa e desenvolvida para se comunicar e expressar-se;

f.3 - Cinestésico: a habilidade que busca ter uma boa coordenação motora;

f.4 - Espacial: habilidade para formar imagens e modelos de realidades espaciais;

f.5 - Musical: Habilidade para seguir músicas e ter boa audição;

f.6 - Interpessoal: Habilidade de empatia e boa relação e contato com outras pessoas;

f.7 - Intrapessoal: Habilidade para conhecer a si mesmo e ser capaz de aproveitar suas potencialidades.

f.8 - Naturalista: A Habilidade para entender e organizar as pautas da natureza.

\section{As escalas de observação.}

Este percurso recorrido pela explicação dos estilos de aprendizagem tem servido de base para o estudo de como pôr em prática melhorias no ensino a partir de como o aluno aprende, o que inexoravelmente passa por criar formas de analisar e estudar diretamente os estilos de aprendizagem dos alunos, seja por meio de inventários, questionários ou escalas.

Para explicar melhor a fundamentação do modelo Registro de Estilo de Aprendizagem -REA- de Sebastian-Heredero (1996), que se apresenta a seguir: 
O inventário de estilos de aprendizagem "Learning Style Inventory (LSI)" de Kolb (1984) é uma ferramenta interessante que ajuda a identificar as características pessoais e a forma como um estudante processa a informação. O mesmo autor expressa que o aprendiz necessita de quatro classes diferentes de capacidades: experiência concreta, observação reflexiva, conceitualização abstrata e experimentação ativa.

O questionário Alonso de estilos de aprendizagem CHAEA, de Alonso, Gallego e Honey (1995), serve para determinar as experiências de cada aluno no que faz referência aos estilos de aprendizagem: Ativo, Reflexivo, Teórico ou Pragmático, por ele usados a partir da observação e registro.

O Inventário de Estilos de Aprendizagem (Learning Style Inventory and Productivity Environmental Preference Survey - LSI++) de Dunn, R.; Dunn, K. Price, G. (1985), parte de um modelo com 21 variáveis que influenciam na maneira de aprender.

O instrumento de determinação do hemisfério dominante (Herrmann's Brain Dominance Instrument - HBDI), de Herrmann, N. (1982), trata de detectar o perfil de combinação de quadrantes, o qual mostra quantos e quais quadrantes são dominantes. A combinação dos quadrantes origina a seguinte distribuição de hemisférios: $A+B=$ Esquerdo; $\mathrm{C}+\mathrm{D}=$ Direito; $\mathrm{A}+\mathrm{D}=$ Cerebral, e $\mathrm{B}+\mathrm{C}=$ Límbico. Consiste de 120 perguntas que permitem identificar as quatro categorias das preferências mentais ou dos estilos de aprendizagem em função do hemisfério dominante.

\section{O Registro de Estilo de Aprendizagem -REA- de $\operatorname{xxxxxxxxxxxxxxxxx~(1996)~}$}

O Registro de Estilo de Aprendizagem (REA) foi publicado no ano 1996, na obra Programas de Diversificação Curricular: da teoria a prática (xxxxxxxxxxxx, 1996) e, desde sua criação, teve duas funções: em primeiro lugar diagnosticar os estilos de aprendizagem que cada aluno utiliza em seu processo de apropriação do conhecimento, no mesmo sentido que Keefe (1988) indica.

O objetivo do mesmo é constatar quais são os preferentes para poder nos servir deles de forma mais eficiente na tarefa cotidiana, a partir das observações que o professor faz sobre um determinado aluno no contexto escolar, mas, além disso, se manifesta, precisamente pelo seu uso escolar, como uma ferramenta que permite começar a trabalhar sobre os estilos não predominantes para desenvolvê-los e ampliar suas possibilidades de aprendizagens e as situações e contextos de ensino. Em definitivo, desenvolver a capacidade de aprendizagem dos alunos. 
Tem um caráter de instrumento de ajuda docente para o conhecimento dos alunos, que se encaixa dentro das estratégias de uma escola inclusiva, para oferecer uma resposta adequada às necessidades de cada um dos diversos alunos e que redunda em novas formas, a partir deste conhecimento, de planejamento didático e de estratégias no processo de ensino/aprendizagem.

Sua apresentação está organizada em cinco alíneas, que fazem referência a:

- Modo de enfrentar aos processos de ensino e aprendizagem;

- Forma de enfrentar as tarefas;

- Relação com os materiais;

- Respostas frente aos agrupamentos;

- Resposta frente aos apoios/ajudas que possa receber.

Nestas alíneas incluem-se registros de observação docente sobre cada aluno em sua singularidade, que nos cercam das teorias anteriormente expostas, nos permitindo ver como os quatro princípios de Kolb (1984) estão incorporados nas formas de se enfrentar as tarefas com as dicotomias apresentadas por Felder e Silverman (1988); a forma de entrada da informação VAK de Bandler e Grinder (1979) aparecem na relação com os materiais; os estímulos que apresentam Dunn, Dunn e Price (1985) estão em diversos tópicos, ou ainda, as teorias de Kohlber (1969), que aparecem nas formas de agrupamento.

Por conseguinte, poderíamos dizer que se trata de um instrumento complexo e holístico que pretende ter um conhecimento amplo. Mas, por sua vez, muito específico para determinar por observação e reflexão um estilo de aprendizagem, a resposta que melhor se adapte às necessidades específicas de cada aluno, desde a perspectiva de que este feito permitirá reduzir as dificuldades de aprendizagem dos alunos e alunas nesta versão revisada e traduzida ao português. 


\section{Registro do estilo de aprendizagem -REApt-}

C) Autores: versão português 2019

Cessão de direitos universal para uso escolar por orientadores, psicopedagogos, coordenadores, professores e gestores, sempre que usada a referência dos autores de forma adequada e não separada do modelo REA

\section{Modo de enfrentar os processos de ensino-aprendizagem}

\subsection{Hábitos de trabalho que manifesta}

Adequados (estuda com frequência no mesmo horário, no mesmo lugar, em iguais condições ambientais...)

Inadequados

\subsection{Condições físicas e ambientais da sala em que melhor se desenvolve}

Luz que prefere

Natural

Artificial

Indistintamente

Nível de ruído que requer em seu trabalho

Necessita de silêncio

É capaz de trabalhar com murmúrios

Pode trabalhar com barulho

Temperatura que é mais cômoda

Calor

Temperatura média

Frio

Hora do dia em que melhor rende na sala

Primeiras horas

Últimas horas

Depois do recreio

Lugar na sala que prefere

Perto do professor

Só/isolado

Nas últimas filas

Junto com um determinado colega

\subsection{Atitude para a aprendizagem}

Positiva (mostra uma predisposição favorável para as tarefas e atividades de estudo)

Normal (realiza as tarefas, ainda que sem excessivo interesse)

Negativa (mostra uma predisposição nada favorável para as tarefas e atividades de estudo, não lhe interessa o trabalho escolar)

1.4. Resistência à frustração (grau em que persiste no esforço, persevera ante as dificuldades $e$ assume os fracassos)

Alta

Média

Baixa

\subsection{Nível de atenção e concentração (intensidade)}

Alta

Média

Baixa

Atende somente determinados estímulos. Especifique quais: 


\subsection{Capacidade de manter a atenção (duração)}

Atende durante um tempo prolongado

Atende durante pouco tempo

Atende um tempo suficiente em determinadas condições. Especifique quais:

\subsection{Tipo de motivação}

Medo do fracasso (trabalha para evitar as críticas dos pais ou professores)

Intrínseca-realização (trabalha pelo interesse que suscita o conteúdo e para fazer bem as coisas.)

Desejo de sobressair (trabalha por desejo de que os demais reconheçam seus êxitos)

\subsection{Atribuição causal}

Atribui o sucesso a:

Capacidade

Esforço

Sorte

Professor benevolente

Tarefa fácil

Outras causas. Especifique:

Atribui o fracasso a:

Falta de capacidade

Falta de esforço

Má sorte

Professor muito exigente

Tarefa difícil

Outras causas. Especifique:

\subsection{Tipo de reforço a que responde}

Elogios

Verbalizações negativas (repreensões)

Menções

Escolha de atividades

Prêmios materiais

Sentar-se com determinados colegas

\subsection{Tipo de estímulos preferidos}

Sociais

Materiais

De atividade

\section{FORMA DE ENFRENTAR AS TAREFAS E ATIVIDADES.}

\subsection{Estilo cognitivo (assinalar um de cada par de itens)}

Analítico - Sintético

Indutivo - Dedutivo

Impulsivo - Reflexivo

Global/holístico - Atomista/local

\subsection{Estratégias de resolução de problemas que utiliza com mais frequência}

Ensaio-erro

Aproximações sucessivas

Trabalhar para trás

Subdividir a tarefa 
Outras. Especifique:

\subsection{Habilidades e técnicas de estudo que utiliza com mais frequência}

Memoriza

Sublinha, resume e faz esquemas

Faz apontamentos durante as explicações em aula

Utiliza técnicas de leitura compreensiva

Reescreve um texto com suas próprias palavras

Elabora representações gráficas para ajudar a compreender (diagramas, quadros sinóticos, mapas conceituais...)

Planeja os processos de estudo e de revisão

Outras. Especifique:

\subsection{Tipo de dificuldades mais frequentes na realização das tarefas}

Dificuldades conceituais (não compreende ou assimila conceitos)

Dificuldades algorítmicas (cálculo, procedimentos padrão)

Dificuldades estratégicas (assinale uma das quatro seguintes):

Não prevê o tempo que vai necessitar

Não planeja as sequências de trabalho (não planeja a tarefa e os passos a seguir)

Não chega ao final das sequências estabelecidas

Não revisa os resultados obtidos

Nenhuma em particular

\section{5. Áreas ou aspectos da aprendizagem que prefere}

Instrumentais (aquisição de destrezas intelectuais básicas: leitura, escrita, cálculo e raciocínio)

Cognitivo-teóricas (estudo de conceitos, teorias...)

Artística

Prático-manipulativas (trabalhos de campo, laboratório...)

Educação Física

Outras. Especifique:

\subsection{Tipo de atividades que prefere}

As que implicam fazer ou realizar uma tarefa

As que implicam observação

As que implicam pensar, imaginar, representar as coisas

\subsection{Modalidade preferida de resposta (quando deve demonstrar o que sabe)}

Oral (prefere dizer, expor, explicar, contestar oralmente)

Escrita (prefere escrever, redigir, elaborar informes)

Manipulativa (prefere desenhar, construir, fabricar, manipular)

Outras (gestual, motora...). Especifique quais:

\subsection{Finalização das tarefas}

Inicia e termina as atividades

Inicia as atividades e as abandona

Não inicia as atividades

\subsection{Atitude perante atividades repetitivas, que já domina}

As realiza com prazer

Fica desanimado

Bloqueia-se e não sabe o que fazer 
As abandona

As rejeita

Mostra-se agressivo

Mostra-se conformista (as faz porque lhe pedem)

Aborrece e abaixa o nível de atenção, surgindo maior número de erros

\subsection{Atitude perante atividades difíceis, distantes de suas capacidades}

As realiza com prazer

Persiste

Bloqueia-se

Desanima-se

As abandona

As rejeita

Mostra-se agressivo

Recorre a procedimentos prévios que domina, porém não avalia os resultados

Desenvolve estratégias de aproximação com o resultado final

Conforma-se com resultados parciais

2.11. Atitude em relação a atividades que representam um desafio (um pouco acima de suas capacidades)

É motivado pelo desafio

Esforça-se

Abandona a atividade

Fica desanimado

Bloqueia-se

Mostra-se agressivo

Recorre a procedimentos prévios que domina, porém não avalia os resultados

Desenvolve estratégias de aproximação com o resultado final

\section{RELAÇÃO COM OS MATERIAIS}

\subsection{Grau de manuseabilidade dos materiais segundo o conteúdo}

Domina o uso de materiais manipulativos ou icônicos (simples)

De maneira autônoma

Com ajuda

De forma insuficiente

Domina o uso de materiais simbólicos ou abstratos (complexos)

De maneira autônoma

Com ajuda

De forma insuficiente

\subsection{Grau de manuseabilidade dos materiais segundo a estrutura}

Domina o uso de materiais muito estruturados

De maneira autônoma

Com ajuda

De forma insuficiente

Domina o uso de materiais pouco estruturados

De maneira autônoma

Com ajuda

De forma insuficiente

\subsection{Tipo de material que prefere}

Manipulativo (objetos manejáveis, tangíveis)

Icônico ou gráfico (imagens, desenhos, slides, diagramas...) 
Auditivo (gravações, explicações...)

Audiovisual (vídeo)

Impresso (livros, apostilas, Xerox...)

3.4. Modalidade sensorial preferida de obter informação (como gosta que lhe ensinem)

Visual (prefere observar, ler)

Auditiva (prefere escutar)

Audiovisual (prefere a combinação de voz e imagem: explicação apoiada por esquemas, vídeo...)

Cinestésica ou manipulativa (prefere que lhe ofereçam objetos que possa tocar, aprender por meio de atividades manipulativas)

\section{Resposta perante os diferentes sistemas de agrupamento}

\subsection{Forma preferida de estudar fora de sala para fazer tarefas ou trabalhos}

Geralmente estuda em grupo

Geralmente estuda individualmente

Busca a ajuda de outras pessoas fora da escola

\subsection{Interação didática que prefere na classe}

Trabalha em grupos grandes com muitos alunos

Trabalha em pequenos grupos com poucos alunos

Trabalha individualmente

Gosta mais da explicação do professor

\subsection{Resposta diante do trabalho em grupos grandes}

Toma iniciativas e aponta sugestões

Realiza as tarefas ainda que não participe ativamente

Resiste em trabalhar, não faz as tarefas

Rejeita as situações em que se trabalha com toda a sala

\subsection{Resposta perante o trabalho em pequenos grupos}

Toma iniciativas e contribui com sugestões

Realiza sem reclamar as tarefas que lhe foram indicadas

Observa sem participar

Ainda que não manifeste rejeição, resiste em trabalhar

Nega-se a trabalhar em grupo

\subsection{Resposta perante o trabalho individual}

Toma iniciativas e contribui com sugestões

Realiza sem reclamar as tarefas que lhe indicaram

Ainda que não manifeste rejeição (por exemplo, escuta as instruções com certa atenção), não intervém nem trabalha, ou o faz depois de muita insistência

Rejeita o trabalho individual

\subsection{Relações em sala de aula (interação observada com seus colegas)}

Colabora e ajuda seus colegas

Mantém interações positivas com seus colegas

É aceito pelos colegas

Costuma permanecer isolado em classe

Perturba a aula (indisciplinado)

É rejeitado na sala 


\section{Providência de apoios para ele estudar e/ou trabalhar.}

\subsection{Compreensão de instruções}

Compreende sem ajuda as instruções complexas

Compreende sem ajuda as instruções simples

Necessita de ajuda para compreender as instruções complexas

Compreende com dificuldade as instruções, ainda que lhe proporcione ajuda

\subsection{Apoios que precisa o aluno para trabalhar}

Guia físico (guia-lo fisicamente nas tarefas)

Guia verbal externo (dizer-lhe o que fazer)

Guia gestual e por observação (indicar-lhe com gestos, proporcionar-lhe indícios, darlhe modelos, mostrar-lhe como fazer algo)

Guia documental ou simbólico (Xerox, desenhos, gráficos, proporcionar-lhe explicações orais ou escritas...)

Autoinstruções (o aluno dá instrução a si mesmo para guiar sua conduta ou ação)

\subsection{Resposta do aluno perante aos apoios}

Aceita com prazer

Mostra-se conformado

Os rejeita

Mostra-se relutante

\section{Outras observações}

AGRADECIMENTOS: Pesquisa financiada pela UFMS-CNPq. Edital UFMS nº. 163/2017.

\section{REFERÊNCIAS}

ALONSO, C. M.; GALLEGO, D. J.; HONNEY, P. Los estilos de aprendizaje.

Procedimientos de diagnóstico y mejora. Bilbao: Ed Mensajero. 6. ed. 1995.

DUNN, R.; DUNN, K. Teaching students through their individual learning styles. Reston, VA: Reston. 1978.

DUNN, R.; DUNN, K.; PRICE, G. E. Learning Styles Inventory (LSI): An Inventory for the Identification of How Individuals in Grades 3 through 12 Prefer to Learn. Lawrence, KS: Price Systems. 1985

FELDER, R. M.; SILVERMAN, L. K. Estilos de aprendizaje y de enseñanza en la educación de ingeniería. Ing. Educación, v. 78, n. 7, p. 674-681, 1988.

GARDNER, H. Inteligencias múltiples. La teoría en la práctica. Barcelona: Paidós.1995

BANDLER, R.; GRINDER, J. Frogs into Princes: Neuro linguistic programming. Moab, UT: Real People Press. 1979. 
HERRMANN, N. The Theory Behind the HBDI and Whole Brain Technology. Forest City, NC: Herrmann International.1982

KEEFE, J. Aprendiendo perfiles de aprendizaje: manual de examinador. Documento que puede ayudarnos a entender mejor todo los relacionado con el aprendizaje. Reston, VA: Asociación Nacional de Principal de la Escuela. 1988.

KOHLBERG, L. Stage and sequence: The cognitive development approach to socialization. In: D. A. Goslin (Ed.). Handbook of socialization theory. Chicago, IL, Rand McNally. 1969. p. 347-480.

KOLB, D. Experiential Learning: Experience As The Source Of Learning And Development. New York, Prentice-Hall. 1984

KRESS, G. R.; VAN LEEUWEN, T. Multimodal discourse: The modes and media of contemporary communication. Londres: Edward Arnold. 2001.

LOZANO, A. Estilos de Aprendizaje y Enseñanza. Un panorama de la estilística educativa. ITESM Universidad Virtual - ILCE. México: Trillas. 2000.

SEBASTIÁN-HEREDERO, E.; AYALA, C.; GALVE, J.L.; CABRERA, J. Los programas de Diversificación Curricular. De la teoría a la práctica. Madrid. Ed CEPE. 1996.

\section{Como referenciar este artigo}

SEBASTIAN-HEREDERO, Eladio. Estilos de aprendizaje. Un modelo de escala de observación docente para el registro de estilo de aprendizaje -REA-. Revista IberoAmericana de Estudos em Educação, Araraquara, v. 14, n. 4, p. 2301-2317, out./dez. 2019. E-ISSN: 1982-5587. DOI: https://doi.org/10.21723/riaee.v14i4.12384

Submetido em: 25/03/2019

Revisões requeridas: 30/04/2019

Aceite em: 25/08/2019

Aprovado em: 02/09/2019 